\title{
Obstructive Sleep Apnoea in Pakistan: A Single Tertiary Care Center Experience
}

Nadia Sultan ${ }^{1}$, Maham Ajmal ${ }^{1}$, Imad-ud-din Saqib ${ }^{1}$, Amen Mobeen ${ }^{2}$, Mobeen Iqbal ${ }^{3}$, Farrukh Mateen ${ }^{4}$, Sohail Naseem ${ }^{5} 5$, Maimoona Siddiqui ${ }^{4}$, Aamir Iftikhar 6

1. Internal Medicine, Shifa International Hospital, Islamabad, PAK 2. Dermatology, Islamic International Medical College (Riphah International University), Rawalpindi, PAK 3. Pulmonary and Critical Care, Maroof International Hospital, Islamabad, PAK 4. Neurology, Shifa International Hospital, Islamabad, PAK 5. Pulmonology, Shifa International Hospital, Islamabad, PAK 6. Pulmonology/Sleep Medicine, Shifa International Hospital, Islamabad, PAK

Corresponding author: Nadia Sultan, nadiasultan54@yahoo.com

\begin{abstract}
Introduction: Considerable interest has been shown in the field of sleep medicine in recent decades. Obstructive sleep apnoea (OSA) is a common condition that remains neglected in most parts of the world. Data are scarce, if any, when it comes to developing countries. We sought to describe the patient population in a single private tertiary care center from such a country.

Materials and Methods: A cross-sectional study that included a total of 203 patients over a five-year period was conducted. Polysomnographic studies were conducted in a dedicated sleep laboratory, under the supervision of sleep physicians. Data were described and analyzed based on clinical and self-reported outcomes, as well as polysomnographic characteristics, and compared them between genders and severity.

Results: With the participants having an average age of 50.84 years and a BMI of $34.7 \mathrm{~kg} / \mathrm{m}^{2}$, the study found that the increase in age and BMI was significantly correlated with an increase in the severity of obstructive sleep apnea in the Pakistani population. There was a significant difference in sleep latency (20.6 $\mathrm{min}$ in women vs. $10.8 \mathrm{~min}$ in men; $\mathrm{p}$-value $=0.001)$ and efficiency $(63.7 \%$ in women vs. $69.8 \mathrm{in} \mathrm{men;}$-value $=$ 0.02 ) between the two genders. Decreases in nadir saturation, total sleep time, and sleep latency were also associated with an increase in the level of severity.
\end{abstract}

Conclusion: There is a dire need for Pakistani, and in extension Asian, medical professionals to ramp up their pace to meet the needs of their population with regard to sleep medicine.

Received 10/05/2019

Review began 12/07/2019 Review ended 12/17/2019 Published 12/24/2019

\section{() Copyright 2019}

Sultan et al. This is an open access article distributed under the terms of the Creative Commons Attribution License CC-BY 3.0., which permits unrestricted use, distribution, and reproduction in any medium, provided the original author and source are credited.
Categories: Internal Medicine, Neurology, Pulmonology

Keywords: sleep, sleep medicine, sleep disorders, obstructive sleep apnea, polysomnography

\section{Introduction}

With the increasing improvements in healthcare and the general standard of living, interest is increasing in conditions that previously had little or no value. Many of these conditions indirectly affect mortality rates by helping in the management of related diseases. Sleep-disordered breathing (SDB) is one of such conditions, whose importance has just been recently recognized [1]. Awareness among physicians, let alone the general population, remains low despite its high prevalence in the general population. When left untreated, SDB has resulted in cardiovascular disease, obesity, diabetes, mental health disorders, reduced work productivity, road traffic accidents, and even death [2-6].

Obstructive sleep apnoea (OSA) comprises a major part of the spectrum of SDB. It is characterized by recurrent episodes of complete or partial obstruction of the upper airway during sleep, resulting in breathing pauses (apnoeas or hypopneas), snoring and frequent awakenings, leading to sleep fragmentation, and eventually excessive daytime sleepiness due to an unrefreshed sleep [7]. At the physiological level, there is insufficient oxygenation and release of stress hormones, which then result in the aforementioned adverse health conditions.

Most of the available data on the prevalence and risk factors of OSA come from studies conducted among whites of European descent, US Hispanics, and African American populations [8]. These studies have suggested and shown differences among racial groups that imply a role of cultural and environmental factors. Despite the lack of data in Asia, the prevalence is reported to be around 2.1\%-7.5\% [9-10]. In Pakistan, studies conducted in Karachi, based on the Berlin questionnaire, revealed that $10 \%-12.4 \%$ of the population has a high risk of OSA [11-12]. Another study conducted in Lahore was intended to describe the characteristics of a small population of 30 patients diagnosed with this condition by formal overnight polysomnography (PSG) [13].

This study attempts to evaluate a large group of Pakistani population diagnosed with OSA, describing its 
demographic, clinical and polysomnographic characteristics, including its associated conditions and risk factors. The study sought to examine the effects of OSA on sleep architecture, the association between OSA and body mass index (BMI), gender influence, and the correlation between severity to clinical and polysomnographic parameters in our population.

\section{Materials And Methods \\ Study design}

The study was a cross-sectional observational study, conducted at Shifa International Hospital after obtaining approval from the Hospital ethics committee and approval from the Institutional Review Board (reference no: 946-221-2018).

\section{Setting}

Individuals who underwent an overnight PSG study from the period of January 2013 to May 2018 at Shifa sleep disorder centre, Islamabad, were included. This is a two-bed sleep laboratory run by a multidisciplinary team of sleep physicians, neurologists, and neurophysiologists. These patients were mainly characterized by symptoms of snoring and/or excessive daytime somnolence, which were advised of this research or presented with some other conditions, but were found to have symptoms suggestive of SDB and eventually referred to the study.

\section{Participants}

All patients who underwent a PSG study within the aforementioned period of time were included. Informed consent was obtained and the procedure was explained before proceeding with the test. Patients completed the questionnaires under the supervision of a well-trained personnel in accordance with the hospital's policy. Patients with incomplete medical records and patients who underwent a second PSG test were excluded from the study.

\section{Operational definitions}

a) OSA is defined by the presence of at least five obstructive respiratory events (apnoeas, hypopnoea, or respiratory effort-related arousals) per hour of sleep during an overnight PSG study. OSA was considered mild when apnoea hypopnoea index (AHI) $\geqslant 5$ and $<15$, moderate when AHI $\geqslant 15$ and $\leqslant 30$, and severe when $\mathrm{AHI}>30$.

b) AHI refers to the number of apnoea plus hypopnoea events per hour of sleep.

c) Apnoea was considered to be greater than $90 \%$ reduction in airflow that lasted for at least $10 \mathrm{~s}$ from baseline, while hypopnoea reduced ventilation by at least $50 \%$, resulting in a $4 \%$ or more decrease in saturation due to partial airway obstruction or event-related arousal.

d) The Sleep Architecture described the various stages of sleep experienced by the participant.

e) Sleep Efficiency was determined by taking the ratio of the total amount of time slept to the total amount of time spent in bed.

f) Continuous Positive Airway Pressure (CPAP) is a device that is titrated according to the Academy of Sleep Medicine (AASM) manual guidelines, which allows the technologist to increase the positive airway pressure throughout the polysomnographic recording in order to determine the single fixed pressure that would eliminate respiratory disturbances.

g) Hypertension is defined as participants on any antihypertensive medication with a physician's prescription of any specialty at the time of study or those who began treatment during the course of the study.

h) BMI was calculated by dividing the body weight $(\mathrm{kg})$ by the square of the height $(\mathrm{m})$. A BMI range of 25 29.9 was considered overweight, and 30 and above as obese.

i) Craniofacial abnormalities comprised a history of nasal obstruction or any other facial anatomical deformity affecting breathing.

j) Cardiovascular Complications encompassed a history of chest pain or discomfort diagnosed as angina by a cardiologist, a history of arrhythmias/heart block or myocardial infarction, or a history of coronary artery stenting, stroke, or transient ischemic attack (TIA) perceived based on information provided by patients through questionnaires.

k) Mallampati Score was assessed on the spot by a physician, asking the patient to open his mouth as wide as 
possible with a full tongue protrusion and no sounds. A standard I to IV grading system was used based on the visibility of the soft palate, the uvula, and the base of the tongue; class I: soft palate, fauces, uvula and visible pillars, class II: soft palate, fauces and visible uvula, class III: soft palate and visible uvula base, and class IV: soft palate not visible at all.

1) Epworth Sleepiness Scale (ESS) > 10 was considered significant for excessive daytime somnolence [14].

m) Berlin Questionnaire : the risk was labeled significant when symptoms mentioned in Sections 1 and 2 were persistent (>3-4 times/week) from the symptoms listed. Section 3 was positive when there was a history of hypertension or BMI $\geqslant 30 \mathrm{~kg} / \mathrm{m} 2$. According to this questionnaire, to be considered as a high risk of sleep apnoea, an individual had to be positive in at least two of the three abovementioned sections [15].

\section{Data sources and measurement}

We sorted baseline data based on the subject's records, such as age, gender, BMI, and comorbid conditions. The self-reported questionnaire based on the Berlin questionnaire, the Mallampati score, and the ESS filled out by subjects before the precedence of the study were also analyzed, while polysomnographic data were retrieved from computer-based software. A standard polygraph, utilizing polysmith software, was used to record the electroencephalogram (EEG), electrocardiogram (ECG), electrooculogram (EOG), electromyogram (EMG) of the chin and bilateral tibialis anterior muscles, airflow measurements, and chest wall and abdominal movements. Airflow was measured using nasal and oral thermistors, and a nasal pressure transducer. Respiratory effort was monitored with piezoelectric belts to measure the movement of the chest and abdomen. Oximetry was measured using a finger probe placed on the index finger. The study used snore microphones to record snoring; these were attached to the neck along with other audio-visual recording devices. All studies were analyzed by trained technicians and sleep physicians using the criteria outlined by the AASM and in concordance with further scoring updates provided by AASM.

\section{Data entry and statistical analysis}

Data entry and analysis were performed using SPSS version 24. After ensuring correct data entry, patient identifiers were removed. Descriptive statistics were used to report age, gender, BMI, symptom presentation, self-reported questionnaire scores (Mallampati, Berlin, and ESS), AHI, and sleep architecture. Subsequently, multivariate analysis was used for the same parameters while controlling other parameters. The p-value of less than 0.05 was considered significant.

\section{Results}

A total of 300 patients with suspected OSA underwent a comprehensive diagnostic PSG in the specified period of time. Of these, 97 patients were excluded on the basis of incomplete records, leaving 203 patients eligible to qualify for the study. There were 134 (66\%) men and 69 (34\%) women with an average age of 50.84 years and a mean BMI of $34.7 \mathrm{~kg} / \mathrm{m} 2$.

\section{Clinical and self-reported questionnaire}

Loud snoring was invariably present in a majority of the patients (63\%). Daytime sleepiness was the second most commonly reported symptom (25\%), and the mean ESS score was 13.7 . The majority of patients (64.6\%) were positive in all three categories of the Berlin questionnaire, with $32.2 \%$ being positive in two categories.

Three-quarters of the patients (74\%) complained of other symptoms as well, in association with or without the two most common symptoms mentioned earlier. These included morning headaches and fatigue. The history of addiction is mainly related to smoking (9.6\%). OSA severity was correlated with baseline characteristics and found a robust relationship between OSA severity and BMI; BMI > 30 was itself an independent predictor of OSA severity ( $\mathrm{p}$-value $=0.05$ ). Age, in general, appears to have a statistically significant effect on OSA severity ( $\mathrm{p}$-value $<0.000$ ). Table 1 describes baseline patient characteristics, including risk factors. 


\section{Cureus}

\begin{tabular}{|c|c|c|c|}
\hline Baseline characteristics & Mean & Range & Standard deviation \\
\hline Age & 50.8 & $13-90$ & \pm 13.4 \\
\hline BMI & 34.7 & $18.5-50$ & \pm 7.0 \\
\hline Epworth sleepiness score & 12.5 & $0-24$ & \pm 6.07 \\
\hline Witnessed apnoeas ${ }^{\star}$ & $71.3 \%$ & Not applicable & Not applicable \\
\hline Symptoms other than LS and EDS ${ }^{\star}$ & $74 \%$ & Not applicable & Not applicable \\
\hline Nasal obstruction* & $37 \%$ & Not applicable & Not applicable \\
\hline Smoking ${ }^{*}$ & $7 \%$ & Not applicable & Not applicable \\
\hline $\mathrm{DM}^{*}$ & $28 \%$ & Not applicable & Not applicable \\
\hline HTN $^{*}$ & $56 \%$ & Not applicable & Not Applicable \\
\hline Cardiovascular complications ${ }^{*}$ & $18.2 \%$ & Not applicable & Not applicable \\
\hline Other diseases presen & $80.0 \%$ & Not applicable & Not applicabl \\
\hline
\end{tabular}

\section{TABLE 1: Baseline characteristics of patients along with risk factors.}

$\mathrm{BMI}=$ body mass index, OSA = obstructive sleep apnoea, $\mathrm{LS}=$ loud snoring, EDS = excessive daytime sleepiness, DM = diabetes mellitus, HTN = hypertension, *percentage of subjects reported on self-reported questionnaire

\section{Polysomnographic characteristics}

With regard to OSA severity, $14.2 \%$ of the patients had mild severity, $14.7 \%$ had moderate, while $50.7 \%$ had severe OSA and $17.7 \%$ of patients did not even qualify as mild OSA. The mean CPAP requirement was 11.7 $\mathrm{cm}$ of water. Table 2 describes the PSG characteristics.

\begin{tabular}{|c|c|c|c|}
\hline PSG characteristics & Mean & Standard deviation & Range \\
\hline Total sleep time (min) & 173 & \pm 115 & $(5-695)^{\star}$ \\
\hline Sleep latency & 14.0 & \pm 19 & $(0.06-147)$ \\
\hline Sleep efficiency (\%) & 67.4 & \pm 18 & $(5-97)$ \\
\hline Nadir saturation & 75.3 & \pm 14 & $(50-97)$ \\
\hline $\mathrm{AHI}$ & 41.0 & \pm 37 & $(0-167)$ \\
\hline NREM 1 (\% of TST) & 21 & \pm 18 & $(0-100)$ \\
\hline NREM 2 (\% of TST) & 64.0 & \pm 20 & $(0-210)$ \\
\hline NREM 3 (\% of TST) & 3.7 & \pm 7 & $(0-32)$ \\
\hline REM (\% of TST) & 10.3 & \pm 10.6 & $(0-53)$ \\
\hline
\end{tabular}

\section{TABLE 2: Baseline PSG characteristics of patients.}

$\mathrm{PSG}=$ polysomnography, $\mathrm{min}=$ minutes, $\mathrm{AHI}=$ apnoea hypopnoea index, $\mathrm{NREM}=$ non-rapid eye movement, $\mathrm{TST}=$ total sleep time, $\mathrm{REM}=$ rapid eye movement, *includes split night and diagnostic studies

\section{Comparison by gender}

Women undergoing PSG were, on average, older compared to men and had a higher BMI. They were also more likely to suffer from comorbid conditions (diabetes mellitus, hypertension) and were more likely to have complaints of daytime somnolence and witnessed apnoea.

We did not notice any significant difference with regards to the three scoring methods (Mallampati, Berlin 


\section{Cureus}

questionnaire scores, and ESS) between the two genders. However, during PSG, significant differences were observed for sleep latency and sleep efficiency. Table 3 details the data comparisons between the two genders, along with significant and nonsignificant differences.

\begin{tabular}{|c|c|c|c|c|}
\hline & Statistics & remales & Males & value \\
\hline \multicolumn{5}{|l|}{ Severity: } \\
\hline 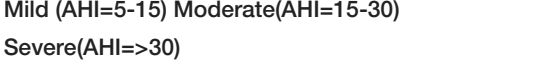 & Number & $8(11 \%) 13(18 \%) 33(47 \%)$ & $21(15 \%) 17(12 \%) 75(55 \%)$ & $0.93^{\star}$ \\
\hline BMI & Mean & 37.9 & 33.1 & $<0.000$ \\
\hline Age (years) & Mean & 55.9 & 48.2 & $<0.000$ \\
\hline Presence of DM & Percentage & $39 \%(\mathrm{~N}=27)$ & $22.7 \%(\mathrm{~N}=30)$ & $<0.000$ \\
\hline Presence of HTN & Percentage & $73 \%(\mathrm{~N}=51)$ & $47 \%(\mathrm{~N}=67)$ & $<0.000$ \\
\hline Presence of nasal obstruction & Percentage & $29.4 \%(\mathrm{~N}=20)$ & $41.3 \%(\mathrm{~N}=55)$ & 0.057 \\
\hline Daytime somnolence & Percentage & $91.4(\mathrm{~N}=63)$ & $78 \%(\mathrm{~N}=104)$ & 0.014 \\
\hline Witnessed apnoea & Percentage & $82 \%(\mathrm{~N}=57)$ & $65.7 \%(\mathrm{~N}=87)$ & 0.030 \\
\hline Epworth sleepiness score & Mean & 13.5 & 13.9 & 0.76 \\
\hline $\begin{array}{l}\text { Berlin questionnaire one category two categories } \\
\text { three categories }\end{array}$ & $\begin{array}{l}\text { Percentage } \\
\text { +ve }\end{array}$ & $\begin{array}{l}3 \%(\mathrm{~N}=2) 19.4 \%(\mathrm{~N}=13) \\
74.5 \%(\mathrm{~N}=50)\end{array}$ & $\begin{array}{l}1.5 \% \quad(\mathrm{~N}=2) 34.8(\mathrm{~N}=45) \\
61.2 \%(\mathrm{~N}=80)\end{array}$ & 0.147 \\
\hline
\end{tabular}

\section{TABLE 3: Data comparison and analysis according to gender.}

BMI: body mass index, DM: diabetes mellitus, HTN: hypertension, N: total number, min: minutes, \%: percentage, NREM: non-rapid eye movement, REM: rapid eye movement. *between moderate and severe group

\section{Comparison by severity}

Although gender and the presence of comorbid conditions had no effect in determining the severity of OSA, there was a significant concordant increase in severity with increasing BMI (p-value $<0.000)$. Patients with increased severity were more likely to have advanced age, daytime somnolence, higher Berlin questionnaire, Mallampati, and ESS. In addition, during PSG, decreases in nadir saturation, total sleep time and sleep latency were associated with an increase in the level of severity. Table 4 compares data based on different levels of severity. 


\section{Cureus}

\begin{tabular}{|c|c|c|c|c|c|c|}
\hline & Statistics & Normal $\mathrm{N}=\mathbf{3 6}$ & Mild $\mathrm{N}=29$ & Moderate $\mathrm{N}=30$ & Severe $\mathrm{N}=108$ & $\begin{array}{l}\mathrm{p} \\
\text { value }\end{array}$ \\
\hline \multicolumn{7}{|l|}{ Gender } \\
\hline Male Female & Number & 2115 & 218 & 1713 & 7533 & 0.36 \\
\hline Age (years) & Mean & 44 & 52.1 & 52.7 & 52.1 & 0.017 \\
\hline BMI & Mean & 31.3 & 31.7 & 35.9 & 36.3 & $<0.000$ \\
\hline Presence of DM & $\begin{array}{l}\text { Percentage } \\
\text { +ve }\end{array}$ & $3 \%$ & $2 \%$ & $5.9 \%$ & $16 \%$ & 0.12 \\
\hline Presence of HTN & $\begin{array}{l}\text { Percentage } \\
\text { +ve }\end{array}$ & $6 \%$ & $8.3 \%$ & $8 \%$ & $32 \%$ & 0.20 \\
\hline Presence of nasal obstruction & $\begin{array}{l}\text { Percentage } \\
+v e\end{array}$ & $4 \%$ & $5.4 \%$ & $4.9 \%$ & 22.2 & 0.29 \\
\hline Daytime somnolence & Mean \% & 69 & 72.4 & 86.6 & 88.7 & 0.028 \\
\hline Witnessed apnoea & $\begin{array}{l}\text { Percentage } \\
\text { +ve }\end{array}$ & 58 & 68.9 & 70 & 76.6 & 0.27 \\
\hline Epworth sleepiness score & Mean & 8.5 & 9.5 & 10.7 & 15.0 & $<0.000$ \\
\hline $\begin{array}{l}\text { Berlin questionnaire one category two } \\
\text { categories three categories }\end{array}$ & $\begin{array}{l}\text { Percentage } \\
\text { +ve }\end{array}$ & $\begin{array}{c}1.5(\mathrm{~N}=3) 20 \% \\
(\mathrm{~N}=12) 12 \%(\mathrm{~N}=16)\end{array}$ & $\begin{array}{c}100(\mathrm{~N}=1) 18 \% \\
(\mathrm{~N}=11) 11 \%(\mathrm{~N}=15)\end{array}$ & $\begin{array}{l}0(\mathrm{~N}=0) 15 \% \\
(\mathrm{~N}=9) 15 \%(\mathrm{~N}=20)\end{array}$ & $\begin{array}{l}0(\mathrm{~N}=0) 44 \% \\
(\mathrm{~N}=26) 60 \% \\
(\mathrm{~N}=79)\end{array}$ & 0.009 \\
\hline Malampati score 1234 & $\begin{array}{l}\text { Percentage } \\
\text { +ve }\end{array}$ & 9128.112 & 1318102.5 & 2124107.6 & 26457076.9 & 0.002 \\
\hline Total sleep time (min) & Mean & 300 & 175 & 158 & 136 & $<0.000$ \\
\hline Sleep latency (min) & Mean & 26.8 & 12 & 11.2 & 11.3 & $<0.000$ \\
\hline Sleep efficiency & Mean $\%$ & 73 & 72.1 & 65.3 & 65.9 & 0.092 \\
\hline Nadir saturation & Mean \% & 83 & 80.0 & 77.9 & 70.0 & $<0.000$ \\
\hline NREM stage 1 & Mean $\%$ & 14.5 & 18.2 & 22.5 & 23.5 & 0.0 \\
\hline
\end{tabular}

\section{TABLE 4: Data comparison and analysis according to severity.}

$\mathrm{BMI}=$ body mass index, $\mathrm{DM}=$ diabetes mellitus, $\mathrm{HTN}=$ hypertension, $\mathrm{N}=$ total number, $\min =$ minutes, $\%=$ percentage, $\mathrm{NREM}=$ non-rapid eye movement, $\mathrm{REM}=$ rapid eye movement

\section{Discussion}

We noted that a middle-aged overweight man is a typical OSA patient in our population. A woman with OSA is usually of an older age and BMI, suffers from other comorbidities, and is more aware of her symptoms before presentation. Severity in both genders increases with increasing BMI and age, a greater report of the symptoms, greater scores in the questionnaires, and a decrease in nadir saturation during sleep study.

Previously, extensive research has been done with regard to the characteristics of multiple populations in relation to OSA, most of which are whites of European descent, US Hispanics and African Americans, and to a limited extent among other populations $[7,10,13]$. A general male predominance has been observed in some studies, ranging from less than 2.3:1 to 7:1 [1]. Our data shows a similar ratio of 2:1.

Young et al. described that those with breathing pauses were three or four times more likely to have AHI $>15$ [1]. However, our study does not support any such link that associate a witnessed apnoea with OSA severity. The median age of women was 55.9 years, indicating that postmenopausal status in our population may suggest a possible role for menopause in SDB.

Studies describe a direct relationship between age and BMI with OSA severity, and an overall greater BMI observed among women [16]. The study also noticed an increase in severity with increasing BMI, along with 
the aforementioned gender differences. Our study showed a significant relationship between OSA severity and age.

Samson et al. and Tasali et al. demonstrated the presence of comorbid conditions (hypertension, type 2 diabetes mellitus, congestive heart failure, and metabolic syndrome) to be positively correlated with OSA severity [17-18]. This study failed to illustrate any of such relationship in our population. However, we noted that women diagnosed with OSA in our population were more likely to have comorbid conditions, possibly due to higher ranges of BMI reported alongside. However, our study highlighted another finding, which states that subjects with severe OSA have a four-fold increased risk of having hypertension when compared with moderate levels (32\% vs. $8 \%$ ). This is consistent with the views of Nieto et al. suggesting that there is a greater probability of arterial hypertension with an increased severity of OSA [19].

It is postulated that the Asian population has distinct craniofacial features based on cephalometric data that predispose them to SDB in a relatively low range of BMI in OSA patients [20]. A third of our population falls into this category in the form of nasal obstruction.

Witnessed apnoeas and daytime somnolence were more frequently reported by women, a finding that has also been observed previously [21]. A survey carried out in the last decade indicated that men and women respond to sleepiness questions differently [22]. Other studies, including our study, question the validity of such claims with results demonstrating that men and women perceive differently, thereby responding differently to the subjective sleep outcomes [22]. This difference could be eliminated using objective measures. However, in our study, both subjective and objective measures yielded similar results.

Tan et al. only recently in 2017, pointed out that the Berlin questionnaire may be useful as a screening tool in the general population due to its high sensitivity (76.9\%) and its negative predictive value (96.3\%) [23]. The study data show a significant correlation of OSA severity with the Berlin questionnaire, Mallampati score, and ESS. Unfortunately, due to lack of a control group, we were unable to determine its use as a screening tool for our population.

O'Connor et al. observed that the rapid eye movement (REM) difference was greater in women than in men with OSA of all degrees of severity [24]. The current study failed to depict any REM duration difference between the two genders.

Some studies suggest that the extent of nocturnal hypoxemia is a direct determinant of OSA severity [25]. Our study confirms this link, which can also further influence daytime symptoms and play a role in increasing blood pressure and the risk of cerebrovascular and cardiovascular events.

Krishnan and Collop noted better sleep efficiency in women compared to men for the same OSA severity, which is also supported by our study [26]. This may be explained by neurochemical mechanisms regulating sleep and wake patterns that are sensitive to the effects of estradiol [27]. However, the exact role of estrogen in modulating sleep patterns in the brain remains unknown.

Kim et al. advocated that short sleep duration $(<5 \mathrm{~h}$ ) has a higher likelihood of increased AHI and visceral obesity [28]. This finding is also reflected in our study, as more time taken to sleep was linked to increased OSA severity; greater time taken to sleep implies shorter sleep duration.

\section{Strengths and limitations}

Even though our study includes the largest number of patients to date of this particular population, the sample size is still a major limitation, rendering many analyses difficult and many difficult-to-explore relationships. An example of this is the deleterious effects of SDB in the cardiovascular state. Only $18 \%$ of our patients had cardiovascular complications in the form of congestive heart failure (CHF), ischemic heart disease (IHD), or stroke; hence it was difficult to establish any statistically significant relationship.

All patient populations included were from a private tertiary care hospital. In a developing country like ours, the majority of the population is faced with affordability issues undergoing lifesaving procedures in such environments, let alone those that improve the quality of life. However, until the moment public hospitals can cater to such facilities, the brunt of such diseases is left for the private sector to bear and therefore, private sector patients constitute the bulk of diagnosed OSA patients.

With the return of foreign-trained medical specialists in low- and middle-income countries, medical facilities are being developed further. At the same time, the lack of sufficient assisting personnel results in disparity between the services provided by the same individual in his trained country and in his country of origin. This may also be due to increased burden on patient in that particular specialty or immaturity on the part of organizations or individuals overseeing quality control or a combination of both. This is evident from the observation that we excluded almost one-third of the total patients in the selected period of time due to incomplete records. The low quality of data is also a reason for the use of relatively vague criteria to describe comorbid conditions in our studies rather than the standard diagnostic criteria. 


\section{Conclusions}

In short, this study describes epidemiology of OSA through polysomnographic data. OSA is a prevalent disorder in Pakistani population causing significant morbidity in both genders. Sleep-related disorders should be taught at all levels of medical education. The increased awareness in public and general practitioners can help in early recognition and timely referral to sleep specialists. We hope that this study will highlight the importance of further research in sleep-related disorders in the Pakistani population.

\section{Additional Information \\ Disclosures}

Human subjects: Consent was obtained by all participants in this study. Shifa International Hospital Ethics committee and Institutional Board issued approval : 946-221-2018. Animal subjects: All authors have confirmed that this study did not involve animal subjects or tissue. Conflicts of interest: In compliance with the ICMJE uniform disclosure form, all authors declare the following: Payment/services info: All authors have declared that no financial support was received from any organization for the submitted work. Financial relationships: All authors have declared that they have no financial relationships at present or within the previous three years with any organizations that might have an interest in the submitted work. Other relationships: All authors have declared that there are no other relationships or activities that could appear to have influenced the submitted work.

\section{Acknowledgements}

Authors thank Mohammad Waseem, a Sleep lab technician at Shifa sleep lab, who provided assistance in data collection and Miss Kholood Janjua, a statistician at Shifa clinical research center for her assistance in statistical analysis.

\section{References}

1. Young T, Palta M, Dempsey J, Skatrud J, Weber S, Badr S: The occurrence of sleep-disordered breathing among middle-aged adults. N Engl J Med. 1993, 29:1230-1235. 10.1056/NEJM199304293281704

2. Peppard PE, Szklo-Coxe M, Hla KM, Young T: Longitudinal association of sleep-related breathing disorder and depression. Arch Intern Med. 2006, 18:1709-1715. 10.1001/archinte.166.16.1709

3. Somers VK, White DP, Amin R, et al.: Sleep apnea and cardiovascular disease: an American Heart Association/American College of Cardiology Foundation scientific statement from the American Heart Association Council for high blood pressure research professional education committee, council on clinical cardiology. J Am Coll Cardiol. 2008, 52:686-717. 10.1016/j.jacc.2008.05.002

4. Marshall NS, Wong KK, Liu PY, Cullen SR, Knuiman MW, Grunstein RR: Sleep apnea as an independent risk factor for all-cause mortality: the Busselton health study. Sleep. 2008, 31:1079-1085. 10.5665/sleep/31.8.1079

5. Young T, Finn L, Peppard PE, et al.: Sleep disordered breathing and mortality: eighteen-year follow-up of the Wisconsin sleep cohort. Sleep. 2008, 31:1071-1078. 10.5665/sleep/31.8.1071

6. Findley LJ, Fabrizio M, Thommi G, Suratt PM: Severity of sleep apnea and automobile crashes. N Engl J Med. 1989, 320:868-869. 10.1056/NEJM198903303201314

7. Epstein LJ, Kristo D, Strollo PJ, et al.: Clinical guideline for the evaluation, management and long-term care of obstructive sleep apnea in adults. J Clin Sleep Med. 2009, 15:263-276.

8. Loredo JS, Soler X, Bardwell W, Ancoli-Israel S, Dimsdale JE, Palinkas LA: Sleep health in US Hispanic population. Sleep. 2010, 33:962-967. 10.1093/sleep/33.7.962

9. Lam B, Lam DC, Ip MS: Obstructive sleep apnoea in Asia. Int J Tuberc Lung Dis. 2007, 11:2-11.

10. Amra B, Farajzadegan Z, Golshan M, Fietze I, Penzel T: Prevalence of sleep apnea-related symptoms in a Persian population. Sleep and Breathing. 2011, 15:425-429. 10.1007/s11325-010-0353-4

11. Taj F, Aly Z, Arif O, Khealani B, Ahmed M: Risk for sleep apnea syndrome in Pakistan: a cross-sectional survey utilizing the Berlin questionnaire. Sleep and Breathing. 2009, 13:103-106. 10.1007/s11325-008-02208

12. Taj F, Aly Z, Kassi M, Ahmed M: Identifying people at high risk for developing sleep apnea syndrome (SAS): a cross-sectional study in a Pakistani population. BMC Neurol. 2008, 8:50. 10.1186/1471-2377-8-50

13. Khan A, Javed N, Saleem K: Obstructive sleep apnea: a review of thirty patients at Fatima memorial hospital Pak J Med Health Sci. 2014, 8:237-242.

14. American Thoracic Society - Epworth Sleepiness Scale (ESS) . (2019). http://www.thoracic.org/members/assemblies/assemblies/srn/questionaires/ess.php.

15. American Thoracic Society - Berlin questionnaire . (2019). http://www.thoracic.org/members/assemblies/assemblies/srn/questionaires/berlin-questionnaire.php.

16. Bixler EO, Vgontzas AN, Lin HM, Have TT, Rein J, Vela-Bueno A, Kales A.: Prevalence of sleep-disordered breathing in women: effects of gender. Am J Respir Crit Care Med. 2001, 163:608-613. 10.1164/ajrccm.163.3.9911064

17. Samson P, Casey KR, Knepler J, Panos RJ: Clinical characteristics, comorbidities, and response to treatment of veterans with obstructive sleep apnea, Cincinnati veterans affairs medical center, 2005-2007. Prevent Chronic Dis. 2012, 9:E46. 10.5888/pcd9.110117

18. Tasali E, Mokhlesi B, Cauter EV: Obstructive sleep apnea and type 2 diabetes: interacting epidemics. Chest. 2008, 133:496-506. 10.1378/chest.07-0828

19. Nieto FJ, Young TB, Lind BK, et al.: Association of sleep-disordered breathing, sleep apnea, and hypertension in a large community-based study. J Am Med Assoc. 2000, 283:1829-1836. 


\section{Cureus}

10.1001/jama.283.14.1829

20. Hsu PP, Tan AK, Chan YH, Lu PK, Blair RL: Clinical predictors in obstructive sleep apnoea patients with calibrated cephalometric analysis - a new approach. Clin Otolaryngol. 2005, 30:234-241. 10.1111/j.13652273.2005.00983.x

21. Larsson L, Lindberg A, Franklin K, Lundback B: Gender differences in symptoms related to sleep apnea in a general population and in relation to referral to sleep clinic. Chest. 2003, 124:204-211.

10.1378/chest.124.1.204

22. Baldwin CM, Kapur VK, Holberg CJ, Rosen C, Nieto FJ: Associations between gender and measures of daytime somnolence in the Sleep Heart Health Study. Sleep. 2004, 15:305-311. 10.1093/sleep/27.2.305

23. Tan A, Yin JD, Tan LW, van Dam RM, Cheung YY, Lee CH: Using the Berlin questionnaire to predict obstructive sleep apnea in the general population. J Clin Sleep Med. 2017, 15:427-432. 10.5664/jcsm.6496

24. O'Connor CH, Thornley KS, Hanly PJ: Gender differences in the polysomnographic features of obstructive sleep apnea. Am J Respir Crit Care Med. 2000, 161:1465-1472. 10.1164/ajrccm.161.5.9904121

25. de Castro JR, Huamaní C, Escobar-Cordoba F, Liendo C: Clinical factors associated with extreme sleep apnoea [AHI> 100 events per hour] in Peruvian patients: a case-control study-a preliminary report. Sleep Sci. 2015, 8:31-35. 10.1016/j.slsci.2015.03.002

26. Krishnan V, Collop N: Gender differences in sleep disorders. Curr Opin Pulmonary Med. 2006, 12:383-389. 10.1097/01.mcp.0000245705.69440.6a

27. Fanfulla F, Grassi M, Eugenia Taurino A, D'Artavilla Lupo N, Trentin R: The relationship of daytime hypoxemia and nocturnal hypoxia in obstructive sleep apnea syndrome. Sleep. 2008, 31:249-255. 10.1093/sleep/31.2.249

28. Kim N, Lee S, Eun C, et al.: Short sleep duration combined with obstructive sleep apnea is associated with visceral obesity in Korean adults. Sleep. 2013, 36:723-729. 10.5665/sleep.2636 